\title{
Kava for the treatment of generalised anxiety disorder (K-GAD): study protocol for a randomised controlled trial
}

Karen M. Savage ${ }^{1,2^{*}}$, Con K. Stough ${ }^{2}$, Gerard J. Byrne ${ }^{3}$, Andrew Scholey ${ }^{2}$, Chad Bousman ${ }^{2,4,5,6}$, Jenifer Murphy ${ }^{1}$, Patricia Macdonald ${ }^{3}$, Chao Suo $^{7}$, Matthew Hughes ${ }^{8}$, Stuart Thomas ${ }^{9}$, Rolf Teschke ${ }^{10}$, Chengguo Xing ${ }^{11}$ and Jerome Sarris ${ }^{1,2}$

\begin{abstract}
Background: Generalised anxiety disorder (GAD) is a chronic and pervasive condition that generates high levels of psychological stress, and it is difficult to treat in the long term. Current pharmacotherapeutic options for GAD are in some cases only modestly effective, and may elicit undesirable side effects. Through targeted actions on the gamma-aminobutyric acid (GABA) pathway, the South Pacific medicinal plant kava (Piper methysticum) is a non-addictive, non-hypnotic anxiolytic with the potential to treat GAD. The evidence for the efficacy of kava for treating anxiety has been affirmed through clinical trials and meta-analyses. Recent research has also served to lessen safety concerns regarding the use of kava due to hepatotoxic risk, which is reflected in a recent German court overturning the previous kava ban in that country (which may in turn influence a reinstatement by the European Union). The aim of current research is to assess the efficacy of an 'aqueous noble cultivar rootstock extract' of kava in GAD in a larger longer term study. In addition, we plan to investigate the pharmacogenomic influence of GABA transporters on response, effects of kava on gene expression, and for the first time, the neurobiological correlates of treatment response via functional and metabolic imaging.

Methods/Design: This clinical trial is funded by the Australian National Health and Medical Research Council (APP1063383) and co-funded by MediHerb (Integria Healthcare (Australia) Pty. Ltd). The study is a phase III, multi-site, two-arm, 18-week, randomised, double-blind, placebo-controlled study using an aqueous extract of noble kava cultivar (standardised to $240 \mathrm{mg}$ of kavalactones per day) versus matching placebo in 210 currently anxious participants with diagnosed GAD who are non-medicated. The study takes place at two sites: the Centre for Human Psychopharmacology (Swinburne University of Technology), Hawthorn, Melbourne, Australia; and the Academic Discipline of Psychiatry (The University of Queensland) based at the Royal Brisbane and Women's Hospital, Herston, Brisbane, Australia. Written informed consent will be obtained from each participant prior to commencement in the study. The primary outcome is the Structured Interview Guide for the Hamilton Anxiety Rating Scale (SIGH-A). The secondary outcomes involve a range of scales that assess affective disorder symptoms and quality of life outcomes, in addition to the study of mediating biomarkers of response (assessed via genomics and neuroimaging).

(Continued on next page)
\end{abstract}

\footnotetext{
* Correspondence: ksavage@unimelb.edu.au

${ }^{1}$ The University of Melbourne, Department of Psychiatry, The Melbourne

Clinic, Melbourne, Australia

${ }^{2}$ Swinburne University of Technology, Centre for Human

Psychopharmacology, Swinburne, Australia

Full list of author information is available at the end of the article
}

\section{Biomed Central}

(c) 2015 Savage et al. Open Access This article is distributed under the terms of the Creative Commons Attribution 4.0 International License (http://creativecommons.org/licenses/by/4.0/), which permits unrestricted use, distribution, and reproduction in any medium, provided you give appropriate credit to the original author(s) and the source, provide a link to the Creative Commons license, and indicate if changes were made. The Creative Commons Public Domain Dedication waiver (http://creativecommons.org/publicdomain/zero/1.0/) applies to the data made available in this article, unless otherwise stated. 
(Continued from previous page)

Discussion: If this study demonstrates positive findings in support of the superiority of kava over placebo in the treatment of GAD, and also is shown to be safe, then this plant-medicine can be considered a 'first-line' therapy for GAD. Genomic and neuroimaging data may reveal clinical response patterns and provide more evidence of the neurobiological activity of the plant extract.

Trial Registration Information: ClinicalTrials.gov: NCT02219880 Date: 13 August 2014:

Keywords: Protocol, Anxiety, GAD, RCT, Kava, Kavalactones, GABA, Anxiolytic, Nutraceutical

\section{Background}

Generalised anxiety disorder (GAD) is defined through cognitive and somatic symptomatology; involving primarily excessive chronic worry and anticipatory anxiety [1], in addition to a constellation of presentations such as sleep disturbances, restlessness, edginess/irritability, fatigue, concentration difficulties, and muscular tension [2]. GAD is ubiquitous and persistent, usually with early onset and high prevalence of co-morbid affective disorders and substance misuse [3-6]. Unfortunately, available pharmacotherapies have a modest clinical effect $[7,8]$ and may elicit undesirable side effects [9-11], and while psychotherapeutic treatments are found to be efficacious [12, 13], they are of limited utility for some patients [14, 15]. As a result, many GAD sufferers do not receive adequate treatment [16-18], thereby providing an impetus to explore other therapeutic options.

One such option is kava (Piper methysticum), a plant native to the South Pacific, whose roots have been used in traditional medicine in the form of cold-water extractions (non-alcoholic) to treat a range of health conditions, including anxiety, stress, muscular spasms, pain, and menstrual disorders $[19,20]$. The therapeutic effect of kava is based on the six major lipophilic kavalactones, of which kawain and dihydrokawain (see Fig. 1) have the strongest anxiolytic activity [21]. Limbic structures of the brain have previously been suggested as the principal site of kavalactone action [22]. Kavalactones exert their anxiolytic effect through an array of neurobiological activity, primarily from modulation of gamma-aminobutyric acid (GABA) receptors via blockade of voltage-gated sodium ion channels $[23,24]$, reduced excitatory neurotransmitter release via blockade of calcium ion channels $[25,26]$, and enhanced ligand binding to GABA type A receptors [27]. Other neurochemical effects include reversible inhibition of monoamine oxidase B [28], inhibition of cyclooxygenase [29], and reduced neuronal reuptake of dopamine [30] and prefrontal cortex noradrenalin [31]. This noradrenergic effect differentiates the central biobehavioural effects of kava from those of alcohol and benzodiazepines [32], while the combination of GABA modulation and increased noradrenergic activation contributes to feelings of physical relaxation with increased hedonic tone, with no deleterious effects on cognition [33].

\section{Background evidence}

A Cochrane review and meta-analysis of seven randomised clinical trials (RCTs) using kava mono-preparations (60-280 mg kavalactones) for the treatment of generalised anxiety symptoms found a significant reduction of anxiety on the Hamilton Anxiety Rating Scale (HAM-A) for kava compared with placebo $(P=0.01)$ [34]. Similarly, a more recent pooled analysis of six studies using kava versus placebo in the treatment of anxiety symptoms found a significant effect in favour of kava on the HAM-A, with an effect size (Cohen's $d$ ) of 1.1 [35]. Our previous threeweek double-blind, placebo-controlled, crossover RCT $(n=60)$, using a water-soluble rootstock extract of a noble kava cultivar in chronic generalised anxiety [36], involved a preparation standardised to a dose of $250 \mathrm{mg}$ kavalactones. Following a placebo run-in phase there was a reduction of -9.9 points on the HAM-A in the first kava phase versus only a -0.8 point in the first placebo phase, and a reduction in the second crossover phase from kava of -10.3 points compared to a rise of +3.3 points for placebo (Cohen's $d=2.24, P<0.0001$ ).

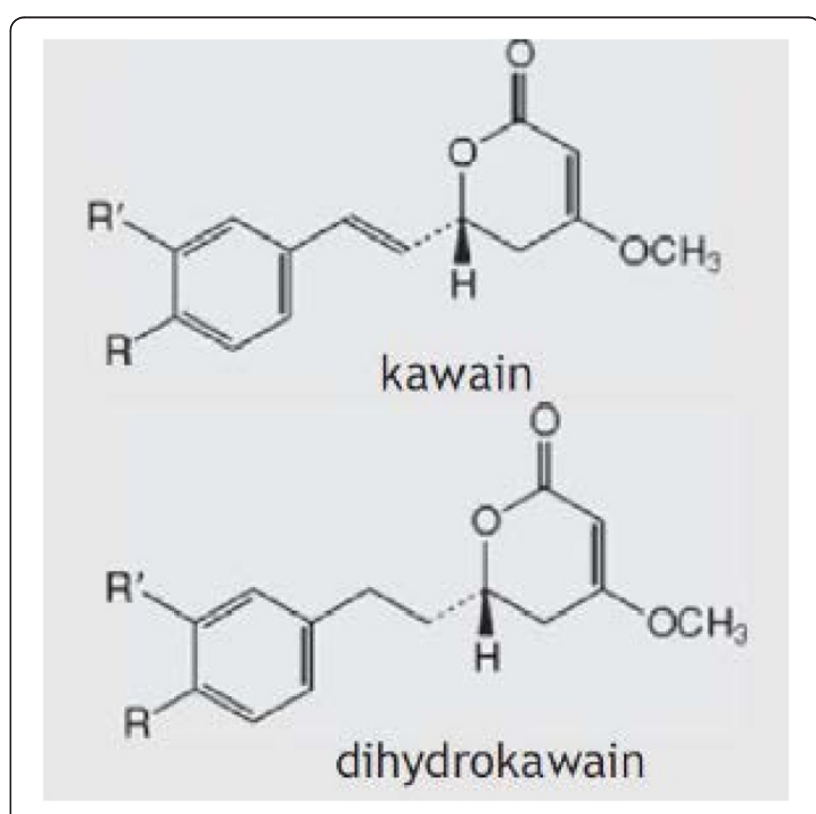

Fig. 1 Active constituents of kava 
In a subsequent six-week parallel double-blind RCT [37] involving 75 participants with diagnosed GAD (58 randomised to $120 \mathrm{mg}$ daily kavalactones titrated to $240 \mathrm{mg}$ for non-response), a group $\times$ time interaction was found $(P=0.046)$ for a significant reduction in HAM-A scores in favour of kava over placebo. Further, kava significantly reduced participant anxiety by -4.2 , representing a moderate effect size (Cohen's $d=0.63$ ). For participants with moderate-tosevere level anxiety (as assessed on the MINI Plus diagnostic interview), the treatment effect was more pronounced $(P=0.020)$, with a larger effect size $(d=0.80)$. The effects were still significant after controlling for baseline Montgomery-Åsberg Depression Rating Scale (MADRS) depression $(P=0.01)$, baseline Beck Anxiety Inventory (BAI) anxiety $(P=0.05)$, thyroid function $(P=0.02)$, and weekly caffeine use $(P=0.03)$. Further sub-analysis of participants with pure GAD and no other DSM-IV diagnosed co-morbid anxiety disorder (panic disorder, social phobia, post-traumatic stress disorder, obsessivecompulsive disorder) revealed a significant group $\times$ time interaction $(P=0.020 ; d=1.28)$, with a reduction of -8.5 points for kava on the HAM-A compared to -2.3 points for placebo. As part of this trial we also examined five GABA transporter polymorphisms as potential pharmacogenetic markers of kava response. We observed a significant monotonic trend for two polymorphisms in which the number of rs2601126 T-alleles $(P=0.021)$ or of rs2697153 A-alleles $(P=0.046)$ was associated with significant reductions in HAM-A scores within the kava group.

\section{Trial objectives}

The primary objective of this trial is to confirm the effectiveness and safety of kava as a pharmacological approach to treating GAD, by conducting a longer term, multicentre RCT with a large sample using a standardised pharmaceutical-grade aqueous rootstock extract of a noble kava cultivar in participants with current diagnosed GAD. As secondary objectives, we also seek to: 1) replicate our previously observed association between genetic variation in the GABA transporter and kava treatment response, 2) elucidate the mechanisms underpinning the efficacy of kava within the GABA and glutaminergic pathways through functional and metabolic neuroimaging, and 3) examine the effects of kava on the expression of selected genes within the GABA, glutamate, dopamine, serotonin, and adrenergic pathways.

\section{Hypotheses}

\section{Primary hypothesis}

1) Kava will be superior to placebo in the treatment of GAD symptoms, assessed by change in Structured Interview Guide for the Hamilton
Anxiety Rating Scale (SIGH-A) [38] scores after the 16-week treatment phase.

\section{Secondary hypotheses}

2) Kava will be superior to placebo on a range of secondary affective disorder outcome scales (Beck Anxiety Inventory [39], Penn State Worry Questionnaire [40], and Montgomery-Åsberg Depression Rating Scale [41]).

3) Kava will be superior to placebo on health-related quality of life measured using The World Health Organization Quality of Life - BREF (WHOQOLBREF) [42], the Social Re-adjustment Rating Scale [43], and the Kessler Psychological Distress Scale [44].

4) Overall sexual satisfaction and performance rated on the Arizona Sexual Experiences Scale [45] will be in favour of kava compared to placebo, in women but not men.

5) Personality trait factors including anxiety, extraversion, socialization, and aggression rated on the Swedish Universities Scales of Personalities (SSP) may be associated with GAD symptomatology and will be measured via this self-reporting measure.

6) Anterior cingulate cortex (ACC) activation and metabolite profiles will be differentially affected in the kava treatment group over placebo from baseline to week 8. Specifically, for ACC metabolite levels, there will be an increase in GABA and decrease in glutamate/glutamine ratio (Glu/Gln); ACC activation: reduction in activation during resting state; ACC activation: reduction in activation associated with anticipatory anxiety in a task-dependent manner. Further, individual differences in rostral ACC activation measured pre-treatment may predict GAD treatment outcomes for kava compared to placebo. Increased levels of activity in the ACC will also be associated with better clinical outcome in GAD participants.

7) Response to kava will be moderated by GABA transporter polymorphisms. Specifically, for every rs2601126-T allele or rs2697153-A allele an individual carries, the greater the reduction of anxiety that will be observed.

8) Individuals in the kava group will have distinct gene expression change profiles compared to the placebo group. Specifically, we hypothesise that expression of GABA pathway genes will be up-regulated following kava treatment and remain stable in the placebo group.

\section{Methods/Design}

\section{Study design and plan}

The design of the study is a phase III, multi-site, two-arm, 18-week, randomised, double-blind, placebo-controlled trial using a standardised pharmaceutical-grade, water- 
soluble extract of kava (240 mg of kavalactones per day) or matching placebo (inert plant-based fibre) in 210 currently anxious participants with GAD. The trial sites are at the Centre for Human Psychopharmacology (Swinburne University of Technology), Hawthorn, Melbourne, Australia and the Academic Discipline of Psychiatry (The University of Queensland) based at the Royal Brisbane and Women's Hospital, Herston, Brisbane, Australia. Recruitment is scheduled to commence September 2015 and through to June 2017 (Fig. 2).

Participants will be required to attend seven visits at the study sites at week 0 (baseline), 2, 4, 8, 12, 16, and 18. At the baseline visit, participants will complete informed consent forms, screening assessments, and mood and anxiety questionnaires (self-reporting and interview-based). Brain imaging component visits (Melbourne site only) are to be scheduled within one week of baseline and week 8 visits.

The study employs an additional two-week singleblind placebo-controlled post-study observation period (that is, for a total trial length of 18 weeks - refer Fig. 3).

All eligible participants will be randomly allocated to a treatment arm, and corresponding treatment will be provided following clearance of blood tests taken at the baseline visit. All subsequent visits will follow the same outline of the baseline session excluding consent forms and screening assessments and including a safety assessment. In addition, participants will be required to provide a blood sample within several days of the baseline, and at week 2,8 , and 16 visits. For female participants not taking hormonemodulating contraception who are otherwise eligible to partake in the brain imaging components, the baseline visit is to be scheduled on days $1-5$ of the menstrual cycle.

\section{Ethics and trial registry}

This study has ethical clearance (Alfred Hospital Research Ethics Committee 137/14, University of Queensland Research Ethics Committee 2014000876,
Swinburne University Human Research Ethics Committee 2014/204), and is registered on ClinicalTrials.gov (protocol number: NCT02219880).

\section{Recruitment}

Participants will be recruited through various advertisement sources:

- A dedicated study website

- Advertisements in local newspapers and radio

- A press release which may involve featured articles in newspapers and appearances by the chief investigator on radio and TV

- Online advertisements, including the Swinburne University 'Participate in Research' webpage (http:// www.swinburne.edu.au/lss/chp/projects/kava.html) and the websites of local anxiety disorder organisations (for example, Anxiety Disorders Association of Victoria, Anxiety Recovery Centre Victoria), Google, and Facebook

- Posters and brochures displayed on campus and in the waiting rooms of local Healthscope pathology centres and medical clinics

\section{Primary outcome measure}

The primary outcome is symptoms of GAD measured by the Structured Interview Guide for the Hamilton Anxiety Rating Scale (SIGH-A), at baseline and subsequent visits up to 18 weeks following randomization. The SIGH-A is a widely used measure of anxious symptomatology in GAD research. It includes measures of persistent worry, anxiety, mood, and somatic symptoms. The SIGH-A has been used in previous studies of pharmacological and psychological treatments for people with GAD. It is reliable (intraclass correlation coefficients for inter-rater reliability of 0.98 and testretest reliability of 0.88 ), has high convergent validity

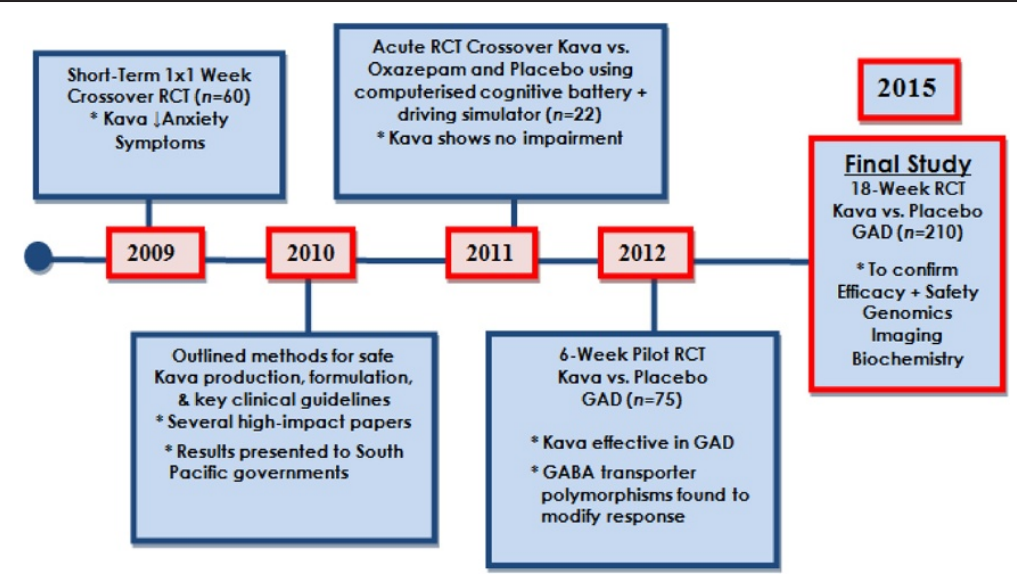

Fig. 2 Kava studies timeline summary 


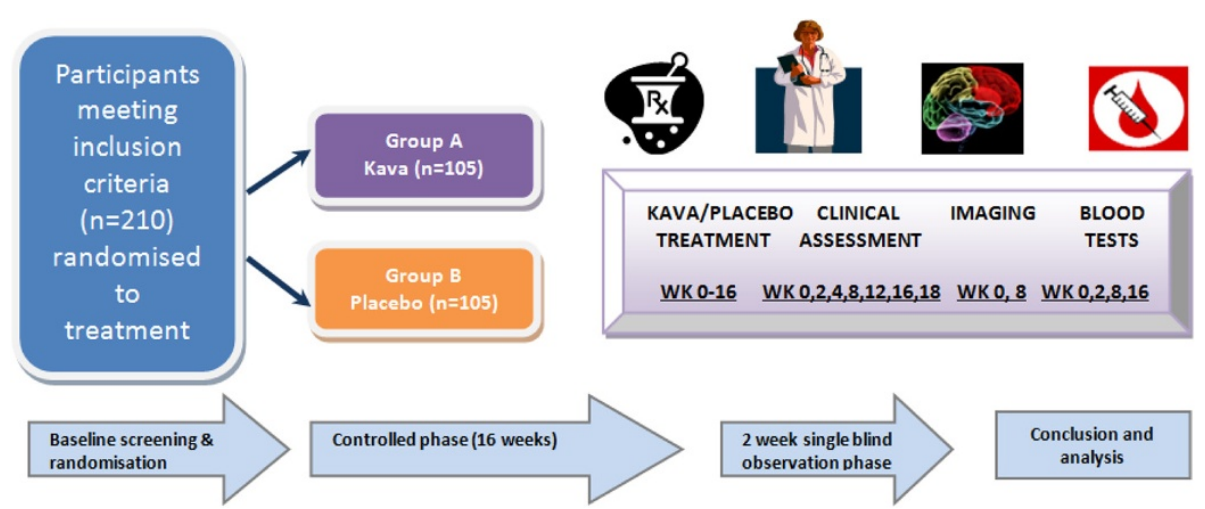

Fig. 3 Clinical trial flowchart

with structured clinical ratings of symptoms of GAD (for example, MINI PLUS 6.0 [46]), and is sensitive to change $[47,48]$.

\section{Secondary outcome measures}

- In addition, the Beck Anxiety Inventory (BAI) will be used as a participant self-report scale to assess anxiety levels. It consists of 21 items ranging from 0 (not present) to 3 (severe). These items are more somatically orientated than the SIGH-A and provide additional information on anxiety outcomes.

- The Penn State Worry Questionnaire (PSWQ) is a 16-item self-report measure of the intensity and excessiveness of worry. Examples of items are "Many situations make me worry" and "Once I start worrying, I cannot stop." Participants rate items on a 5-point Likert scale from 1 ("Not at all typical of me") to 5 ("Very typical of me"). Total scores range from 16 to 80 , with higher scores indicating pathological worry. The PSWQ is widely used and has shown good to very good internal consistency, test-retest reliability, and discriminant validity for GAD versus other anxiety disorders [49].

- Mood will be assessed at each visit using the SIGMA (Structured Interview Guide for the Montgomery-Åsberg Depression Rating Scale (MADRS)), a diagnostic questionnaire which measures severity of depression symptoms. The SIGMA provides structured questions for each item to ensure standardisation of administration. Each item yields a score of 0 to 6 , producing an overall score ranging between 0-60. A higher MADRS score indicates more severe depression. Inter-rater reliability with the SIGMA is reported to be excellent $(r=0.93)$ [50]. The use of this scale is important, as sufferers of GAD often have co-morbid depression, which may affect their response to treatment.
- Quality of life/life stressor measures will be administered at selected visits. The WHOQOL-BREF measure is a reduced version of the original instrument, designed for clinical trial use. It comprises 26 items, covering the domains of physical health, psychological health, social relationships, and environment. The Social Readjustment Rating Scale (SRRS) is a scale using 'Life Change Units' (LCUs) to quantify the number and severity of life stressors occurring in the preceding 12-month period. Both will be administered at baseline and at week 16. The Kessler Psychological Distress Scale (K10) is a 10-item questionnaire designed to obtain a global measure of perceived stress level, with questions pertaining to anxiety and depressive symptoms experienced in the past four-week period. For this study it will be administered at each visit.

- The Arizona Sexual Experiences Scale (ASEX) will be employed to assess the effects of kava on participants' sexual functioning. Specifically, the self-report scale explores effects on desire, arousal level, and physiological responses over the course of the past week. It consists of five self-report items, each rated on a 6-point scale. Total scores can range between 5-30, where higher scores indicate greater sexual dysfunction. The use of this scale is important, as conventional SSRI antidepressant treatments (used to treat GAD) commonly cause sexual side effects, and results of a previous study [37] showed a significant improvement in sex drive for women. If this finding is replicated, it will show a point of difference between kava and conventional treatments. Due to the sensitive nature of the items, completion of this scale is optional.

- The Swedish Universities Scales of Personalities (SSP) is a 91-item self-rated questionnaire based on the Karolinska Scales of Personality (KSP), a scale designed to measure stable personality traits related to psychopathology [51]. It assesses four general temperament dimensions: anxiety, extraversion, socialization, and aggression. This questionnaire will 
be given to participants after their initial baseline visit to complete in their own time and return at the next visit.

- Genotyping of candidate and haplotype-tagging single nucleotide polymorphisms in monoamine, glutaminergic, and GABA transporter genes, measured via blood sample at week 1 , will be achieved using a Sequenom Mass-Array.

- A custom $\mathrm{RT}^{2}$ PCR Array (Qiagen) will be used to examine gene expression of candidate genes (Table 1) at weeks 1 and 8 .

- Anterior cingulate cortical (ACC) region metabolite profiles, including $\mathrm{N}$-acetyl-l-aspartate (NAA), creatine (Crn), glutamate/glutamine (Glu/Gln), and gamma-amino butyric acid (GABA), will be measured using single voxel magnetic resonance spectroscopy (MRS) at weeks 1 and 8 .

- ACC region activity at rest and functional connectivity network (that is, default mode network) will be analysed using resting-state functional magnetic resonance imaging (fMRI) at weeks 1 and 8 .

- ACC region task-dependent activation levels associated with anticipatory anxiety will be assessed using task fMRI (the International Affective Picture System (IAPS; [52]) at weeks 1 and 8.

- Acute anxiety levels related to undergoing scanner session at weeks 1 and 8 will be measured on the Spielberger State-Trait Anxiety Inventory - Trait and State components [53].

\section{Inclusion criteria*}

\section{Aged between $18-70$ years}

2. Meets the DSM-IV and DSM-5 diagnostic criteria for generalised anxiety disorder (GAD) based on structured interview; Mini International Neuropsychiatric Interview-6.0 (MINI 6.0) [54], (note that while the MINI 6.0 uses the DSM-IV criteria, the same criteria are used in the DSM-5)

3. Presents with anxiety (SIGH-A $\geq 18)$ at the time of study entry

4. Fluent in spoken and written English

5. Provides a signed copy of the consent form

*MRI component - Participant eligibility for the imaging component of the study will follow a listing of restrictions typical of imaging safety at the 3-T scanner. All participants will be right-handed, with no metallic implants, piercings, or residue. For females in the imaging component, there will be conditions of enrolment relating to menstrual cycle dates, and they must not be taking hormone-modulating contraception (such as oral contraceptive pills or hormonal implants).

\section{Exclusion criteria}

1. Primary diagnosis other than GAD as determined by the MINI 6.0

2. Presentation of moderate to severe depressive symptoms (MADRS $\geq 18$ ) at time of study entry

3. Presentation of suicidal ideation ( $\geq 3$ on MADRS suicidal thoughts domain) at time of study entry

4. Current diagnosis of a psychotic disorder (for example, schizophrenia) or bipolar I on structured interview (MINI 6.0)

5. Current substance/alcohol use disorder on structured interview (MINI 6.0)

6. Currently taking an antidepressant, mood stabiliser, antipsychotic, anticonvulsant, warfarin or thyroxin, or regularly using a benzodiazepine or opioid-based analgesic (more than two days per week)

7. Current use of St John's wort or contraindicated herbal medication

8. Previous intolerance to kava

9. Three or more failed trials of pharmacotherapy for the current GAD episode

10. Recently commenced psychotherapy (within four weeks of study entry)

11. Known or suspected clinically unstable systemic medical disorder

12. Diagnosed hepato-biliary disease/inflammation

13. Elevated liver enzymes at baseline blood test

14. Pregnancy or breastfeeding, or trying to conceive

15. Not using a medically approved form of contraception (including abstinence) if female and of childbearing age

16. Unable to participate in all scheduled visits, treatment plan, tests, and other trial procedures according to the protocol.

\section{Treatment interventions, randomisation, and blinding}

The active treatment (kava) and placebo product will be identical in colour, size, and shape, bottled and labelled with trial treatment numbers by an independent researcher. The kava is a high-quality noble Borogu cultivar acquired from Southern Pentecost Island, Vanuatu, and manufactured by MediHerb (Integria Healthcare (Australia) Pty. Ltd.). Each tablet is standardised to contain $60 \mathrm{mg}$ of kavalactones with a higher level of kavain and dihydrokavain, and a lower relative level of dihydromethysticin. To ensure replication of odour, a small porous sachet of kava powder is inserted into all treatment bottles. The trial products will be manufactured and stored in accordance with manufacturer instructions, following Pharmaceutical Good Manufacturing Practice.

Participants are assigned study identification numbers sequentially, and treatments are randomised via identification numbers using permutated $3 \times 2$ block randomisation, 
Table 1 Gene expression list

\begin{tabular}{|c|c|}
\hline Gene symbol & Gene name \\
\hline GABBR1 & GABA, B receptor 1 \\
\hline GABBR2 & GABA, B receptor 2 \\
\hline GABRA1 & GABA, A receptor, alpha 1 \\
\hline GABRA2 & GABA, A receptor, alpha 2 \\
\hline GABRA4 & GABA, A receptor, alpha 4 \\
\hline GABRA5 & GABA, A receptor, alpha 5 \\
\hline GABRA6 & GABA, A receptor, alpha 6 \\
\hline GABRB1 & GABA, A receptor, beta 1 \\
\hline GABRB3 & GABA, A receptor, beta 3 \\
\hline GABRD & GABA, A receptor, delta \\
\hline GABRE & GABA, A receptor, epsilon \\
\hline GABRG1 & GABA, A receptor, gamma 1 \\
\hline GABRG2 & GABA, A receptor, gamma 2 \\
\hline GABRG3 & GABA, A receptor, gamma 3 \\
\hline GABRQ & GABA, A receptor, theta \\
\hline GABRR1 & GABA, A receptor, rho 1 \\
\hline GABRR2 & GABA, A receptor, rho 2 \\
\hline ADCY7 & Adenylate cyclase 7 \\
\hline ADORA1 & Adenosine A1 receptor \\
\hline ADORA2A & Adenosine A2a receptor \\
\hline CACNA1A & Calcium channel, voltage-dependent, P/Q type, alpha 1 A subunit \\
\hline CACNA1B & Calcium channel, voltage-dependent, $\mathrm{N}$ type, alpha 1B subunit \\
\hline GNAl1 & G protein, alpha inhibiting activity polypeptide 1 \\
\hline GNAQ & G protein, q polypeptide \\
\hline GPHN & Gephyrin \\
\hline SNCA & Synuclein, alpha \\
\hline NSF & N-ethylmaleimide-sensitive factor \\
\hline $\mathrm{P} 2 \mathrm{R} \times 7$ & Purinergic receptor P2X, ligand-gated ion channel, 7 \\
\hline SLC1A3 & Glial high affinity glutamate transporter \\
\hline SLC32A1 & Solute carrier family 32 (GABA vesicular transporter), member 1 \\
\hline SLC38A1 & Solute carrier family 38, member 1 \\
\hline SLC6A1 & Solute carrier family 6 (neurotransmitter transporter), member 1 \\
\hline SLC6A11 & Solute carrier family 6 (neurotransmitter transporter), member 11 \\
\hline SLC6A12 & Solute carrier family 6 (neurotransmitter transporter), member 12 \\
\hline SLC6A13 & Solute carrier family 6 (neurotransmitter transporter), member 13 \\
\hline ABAT & 4-aminobutyrate aminotransferase \\
\hline ALDH5A1 & Aldehyde dehydrogenase 5 family, member A1 \\
\hline GAD1 & Glutamate decarboxylase 1 \\
\hline GLS & Glutaminase \\
\hline GLUL & Glutamate-ammonia ligase \\
\hline PHGDH & Phosphoglycerate dehydrogenase \\
\hline DRD1 & Dopamine receptor D1 \\
\hline DRD2 & Dopamine receptor D2 \\
\hline DRD3 & Dopamine receptor D3 \\
\hline
\end{tabular}


Table 1 Gene expression list (Continued)

\begin{tabular}{|c|c|}
\hline DRD4 & Dopamine receptor D4 \\
\hline DRD5 & Dopamine receptor D5 \\
\hline COMT & Catechol-O-methyltransferase \\
\hline DBH & Dopamine beta-hydroxylase \\
\hline DDC & Dopa decarboxylase \\
\hline MAOA & Monoamine oxidase A \\
\hline $\mathrm{TH}$ & Tyrosine hydroxylase \\
\hline SLC6A3 & Solute carrier family 6 (dopamine transporter), member 3 \\
\hline SLC6A4 & Solute carrier family 6 (serotonin transporter), member 4 \\
\hline MAOB & Monoamine oxidase B \\
\hline HTR1A & Serotonin receptor $1 \mathrm{~A}$ \\
\hline GRIA1 & Glutamate receptor, ionotropic, AMPA 1 \\
\hline GRIA2 & Glutamate receptor, ionotropic, AMPA 2 \\
\hline GRIA3 & Glutamate receptor, ionotropic, AMPA 3 \\
\hline GRIA4 & Glutamate receptor, ionotropic, AMPA 4 \\
\hline GRIK1 & Glutamate receptor, ionotropic, kainate 1 \\
\hline GRIK2 & Glutamate receptor, ionotropic, kainate 2 \\
\hline GRIK4 & Glutamate receptor, ionotropic, kainate 4 \\
\hline GRIK5 & Glutamate receptor, ionotropic, kainate 5 \\
\hline GRIN1 & Glutamate receptor, ionotropic, N-methyl D-aspartate 1 \\
\hline GRIN2A & Glutamate receptor, ionotropic, $\mathrm{N}$-methyl D-aspartate $2 \mathrm{~A}$ \\
\hline GRIN2B & Glutamate receptor, ionotropic, N-methyl D-aspartate 2B \\
\hline GRIN2C & Glutamate receptor, ionotropic, N-methyl D-aspartate $2 \mathrm{C}$ \\
\hline GRM1 & Glutamate receptor, metabotropic 1 \\
\hline GRM2 & Glutamate receptor, metabotropic 2 \\
\hline GRM3 & Glutamate receptor, metabotropic 3 \\
\hline GRM4 & Glutamate receptor, metabotropic 4 \\
\hline GRM5 & Glutamate receptor, metabotropic 5 \\
\hline GRM6 & Glutamate receptor, metabotropic 6 \\
\hline GRM7 & Glutamate receptor, metabotropic 7 \\
\hline GRM8 & Glutamate receptor, metabotropic 8 \\
\hline ADRA1A & Adrenoceptor alpha 1A \\
\hline ADRA1D & Adrenoceptor alpha 1D \\
\hline ADRA2A & Adrenoceptor alpha 2A \\
\hline ADRB2 & Adrenoceptor beta 2 \\
\hline ADRB3 & Adrenoceptor beta 3 \\
\hline SLC18A2 & Vesicular monoamine transporter \\
\hline SLC6A2 & Norepinephrine transporter \\
\hline CYP3A4 & Cytochrome P450 3A4 \\
\hline CYP2D6 & Cytochrome P450 2D6 \\
\hline CYP2E1 & Cytochrome P450 2E1 \\
\hline$A R$ & Androgen receptor \\
\hline NRG1 & Neuregulin-1 \\
\hline BDNF & Brain-derived neurotrophic factor \\
\hline ABCG2 & Breast cancer resistance protein \\
\hline
\end{tabular}


Table 1 Gene expression list (Continued)

\begin{tabular}{ll}
\hline ABCB1 & P-glycoprotein \\
GAPDH & Reference genes \\
SADH & Reference genes \\
B2M & Reference genes \\
\hline
\end{tabular}

for example, ABAAABABBBA, based on these numbers. Randomisation is performed by staff unrelated to the study. Treatment is allocated sequentially to enrolled participants by trial clinicians who are blinded to treatment coding (with no identifying details on the bottle of which group [A or B] each participant is assigned to, thus providing double-blinding). Participants will take two kava tablets twice per day (total of $240 \mathrm{mg}$ of kavalactones per day) or matching placebo for 16 weeks.

\section{Statistical analyses}

Analysis of data will be conducted with blinding to group allocations. The primary efficacy analysis will assess average treatment group differences for the primary outcome measure (SIGH-A) over the entire study period using a likelihood based mixed-effects model, repeated measures approach (MMRM). Results from the analysis of dichotomous data (for example, demographics and genetic data) will be presented as proportions (for example, relative risks), with $95 \%$ confidence interval, and Fisher's exact test $P$ value where appropriate. Non-parametric statistics will be used when assumptions for parametric methods are violated. Cohen's $d$ effect sizes will be calculated. All tests of treatment effects will be conducted using a two-sided alpha level of 0.05 and $95 \%$ confidence intervals. Data will be analysed using Statistical Package for Social Sciences software (SPSS) [55].

\section{Imaging analysis \\ Imaging data acquisition}

A 3-tesla scanner (Siemens Tim Trio MR scanner) is utilised for the neuroimaging component of the study. Structural MRI: A high-resolution $(1 \times 1 \times 1 \mathrm{~mm})$ T1 weighted scan will be performed. MRS: To measure the level of metabolite concentrations within the region of interest (anterior cingulate cortex (ACC)), single voxel MRS will be applied. PRESS sequence $(\mathrm{TE}=30, \mathrm{TR}=2000$, Ave $=128$, weak water suppression) is first conducted to measure the main metabolism, followed by MEGA-PRESS $(\mathrm{TE}=68, \mathrm{TR}=2000$, suppression frequency $=1.95 \mathrm{ppm}$, Ave $=64)[54,56-58]$ sequence at the identical location to measure concentration of GABA. Finally a waterunsuppressed sequence (16 averages) will be conducted for quantification. Functional MRI: Resting state fMRI (EPI sequence, $\mathrm{TE} / \mathrm{TR}=30 / 2500,3 \times 3 \times 3 \mathrm{~mm}, 33$ slices, $10-\mathrm{min}$ scan acquisitions) will be conducted with the subject's eyes closed in addition to task-based cognitive activation
fMRI study utilising IAPS to elicit anxiety (EPI sequence, $\mathrm{TR}=2000,3 \times 3 \times 3 \mathrm{~mm}, 27 \mathrm{mins}$ ).

\section{Image data analysis}

$M R S$ : LC-model will be used to fit and quantify the concentration of all metabolites (total $\mathrm{N}$-acetyl-l-aspartate, creatine, total choline, myo-inositol, glutamate/glutamine, and gamma-aminobutyric acid (GABA)), after quality control (that is, CLRB $<20 \%$, SNR $>10$ ). Further, partial volume effect will be corrected using high-resolution T1 structural image and in-house voxel co-registration script. Imaging data analysis will be conducted using Statistical Parametric Mapping (SPM) and MATLAB software [59]. Functional MRI: Pre-processing and statistical analysis will be conducted using SPM12 (Wellcome Department of Neurology) and associated toolboxes run on MATLAB [59]. Pre-processing will include slice timing correction, motion correction, co-registration of function and structural data, then non-linear warping into standardised stereotactic space (MNI) and spatial smoothing. Resting-state fMRI will be further band-pass filtered and de-trended, then subjected to correlational connectivity analysis using REST v1.8 (http://www.restfmri.net/ forum/REST_V1.8). The pre-processed task-based fMRI data will be high-pass filter analysed using the general linear model approach in SPM12, from which contrast images of crucial interest will be generated and entered into second level group models. To test the longitudinal effect of treatment group, a flexible factorial design will be used with main effect of time and group, interaction of time $x$ group and covariance. Significant clusters surviving multiple comparison corrections will be reported.

\section{Power analysis}

The study will recruit a sample size of 210 participants (105 participants per arm). The study is powered to detect a small-to-moderate difference between active treatment and placebo on efficacy outcomes. Data will be analysed using data with at least one post-baseline, and all data including baseline measurement, in an intention-to-treat analysis. As such, a conservative effect size F of 0.15 on the primary outcome measure (SIGH-A) for a two-tailed analysis (with alpha $=0.05)$, and the study powered at $80 \%(\mathrm{Z}$ beta $=0.80$ ), with a correlation among repeated measures (analysis of variance (ANOVA) model) over six time points, will require 206 participants (critical $F$ of 3.88). A sample size of 206 (rounded up to 210) will be sufficiently 
powered to provide a statistical difference between active treatment and placebo groups (using the intention-to-treat analysis) with the data.

The sample size of the imaging component of this study is 40 per treatment arm $(n=80)$. Following 12-week supplement administration, a recent MRS study with a sample size of 24 (12 in each arm) detected significant changes in metabolites by $39 \%$ [60]. Our sample size of 40 participants in each arm will provide higher statistical power compared to previous studies, whilst still maintaining sufficient power with a $10 \%$ participant attrition rate, as well as attrition due to unreliable metabolite estimates in one or both time points.

\section{Safety considerations}

While kava is a Therapeutic Goods Association (TGA) listed, over-the-counter nutraceutical product in Australia and has not been shown to cause any confirmed serious adverse reactions in this country, concerns over hepatotoxicity have led to withdrawal or restriction in some other countries. As discussed in the literature [61-63], many reported cases have involved concomitant ingestion of other compounds with potential hepatotoxicity (such as other medications and/or alcohol). At the clinical level, a variety of case study data from patients with kava hepatotoxicity have been gathered, with probable causation reported in a few cases [63]. Previously reported hepatotoxicity issues identified with German kava products may also be due in part to the extraction (ethanol or acetone extraction) method; the use of non-water soluble chemical (ethanol or acetone) extraction techniques (the traditional solvent is water), and use of aerial parts of the plant and root and stem peelings, and poorly prepared, potentially contaminated raw material [63].

In response to safety concerns, the World Health Organization commissioned a report assessing the risk of kava products [64]. Recommendation 2.1.3 from the report suggested that products from water-based suspension preparations be preferentially used over acetonic and ethanolic extracts. The current study addresses these safety concerns by using standardised aqueous formulations of kava from the peeled rootstock of a noble cultivar (such cultivars are higher in kawain and dihydrokavain and lower in dihydromethysticin). Note that in June 2014, the German Federal Institute for Drugs and Medical Devices (Bundesinstitut für Arzneimittel und Medizinprodukte) overturned the previous 2002 ban on the use of kava products. As a European Union regulatory and guideline body, such a change has positive ramifications for the potential reinstatement of kava products based on ongoing empirical evidence from clinical trial research.

\section{Monitoring and treatment compliance}

Study staff involved in all aspects of participant recruitment, assessment, and monitoring hold postgraduate psychology qualifications, undergo training including inter-rater reliability of interviewer scale measures, and are supervised by psychiatrists and medical staff. This study has comprehensive medical supervision. Regardless, the occurrence of an adverse event (AE) may come to the attention of study personnel during study visits and interviews of a study subject presenting for medical care, or upon review of subject data by a study monitor. Any medical condition that is present at the time that the subject is screened for inclusion will not be reported as an AE. However, if it deteriorates at any time during the study, it will be recorded as an AE. All AEs will be graded for severity and relationship to study product. Participants will also be required to complete the Systematic Assessment for Treatment of Emergent Effects (SAFTEE) [65] at each assessment session to assess for AEs. All AEs will be recorded in the adverse event log in the participant case report form (CRF), including the seriousness, severity, and relationship to study product, duration, and outcome. In all cases, researchers will maintain contact with participants who experience an $\mathrm{AE}$ until it has been resolved and symptoms disappear. They will also be asked to notify their general practitioner. If a worsening of a participant's mental state occurs, the study psychiatrist and/or clinical psychologist will be consulted and appropriate steps taken.

Liver function will be assessed pre-treatment, and participants with any abnormal results will be excluded as a safety precaution. In people with healthy liver function, it is not expected that any elevation of serum liver enzyme levels will occur (based on our previous studies using similar standardised pharmaceutical-grade extract of kava). Nevertheless, liver function will be closely monitored via blood samples at regular intervals throughout the study (weeks 2, 8, and 16).

Participants will be required to take the tablets as instructed during the 18 weeks of the study. At each assessment session they will be asked to return all unused tablets, which will be counted for compliance rate (tablet counts will be employed by an independent researcher).

\section{Data and documentation}

Source documents will be kept in the participant files, which will be stored in a locked cabinet in a locked room accessible only to the investigators. Data from study measures will be entered into a password-protected electronic database on a secure network drive and backed up onto a USB/external hard drive stored alongside the participant files in a locked cabinet. All electronic data will be verifiable against source documents. As per Good Clinical Practice guidelines, upon request of regulatory authorities, the principle investigator will make all requested trial- 
related records, including source documents, available for direct access. The study files and all source data will be retained for 15 years from the date of publication of study results, in accordance with university policy. Study protocol and processes have been developed in line with Standard Protocol Items: Recommendations for Interventional Trials (SPIRIT) guidelines [66].

\section{Discussion}

As detailed above in the literature, and through research related to the current project, there is evidence for kava's anxiolytic effect in both subthreshold generalised anxiety and GAD. Due to WHO recommendations to test water-soluble extracts, and the importance of using high-quality rootstock extracts from noble cultivars of kava, further research is required. Although our pilot data are promising, confidence in the use of a watersoluble extract of kava for the treatment of GAD cannot be established without a confirmatory larger, longer term rigorous study. Kava for use in anxiety has many advantages including clinical evidence for efficacy and safety, a relatively low cost, and the general appeal of nutraceutical approaches in the provision of treatment options outside of the conventional medication armamentarium.

Further, examining the neurobiological actions that underpin the anxiolytic effects of kava may contribute to understanding anxiolytic pharmacodynamics within relevant neural pathways, in turn, better informing their use within anxiety disorders. SNPs account for the pharmacodynamics and pharmacokinetics of drug response, and the current study aims to examine the genetic profiles of study participants, and subsequent metabolic and substrate pathway functionality behind differential response to study treatment. Relevant SNPs involved in pathways such as GABA may serve as predictive markers for determining the effectiveness of kava via allelic group in the GAD sample.

The pharmacogenomic component of the study is to be integrated with an investigation of brain metabolites and neurobiological function underpinning response to kava administration.

The interface between the purported anxiolytic properties of kavalactones, the functional and metabolic properties of brain regions associated with the disorder, and the differential response in GAD symptomatology (guided by pharmacogenomic markers) is of particular interest. Several studies have examined kava modulation on neurotransmitters in vitro (for a review see [67]), yet to date no studies have examined in vivo mechanisms of action which may underlie cognitive and physiological effects of kava prescription. Changes to the properties of GABA metabolites as a product of the intervention may be measurable in limbic brain regions in the GAD sample via MRS [54,60,67-70] and could be the key to understanding the neurobiological efficacy of kava administration.

The anterior cingulate cortex's (ACC's) connectivity from lower limbic and hippocampal regions to the prefrontal cortical regions implies a central role in the organisation of affective and cognitive information. The ACC is thought to mediate affective and cognitive aspects of phenomena such as sadness recall, selective attention, loss, error and reward, suppression of negative emotion or cognitions, and autonomic processes that underpin anxiety states [71-79]. As such, (dysfunction within) the ACC may be a principal region underpinning GAD symptomatology, and is therefore the specific region under investigation for changes related to kava ingestion in the current study.

Some limitations to the study design are recognised. First, the design does not include a positive control product such as an SSRI, which may on some measures outperform the active treatment. Second, the use of imaging and genomic testing will need to ensure statistical correction applied for multiple comparisons, and thus the sample size must be reached to ensure adequate power in order to confirm any findings. Third, consideration of the external generalisability of study findings must be given, as the study sample will be 'pure GAD', that is, GAD that is currently untreated without comorbid conditions such as major depression, and with no current symptom treatment regime, either pharmaceutical or psychotherapeutic.

In summary, if this traditional extract of kava is confirmed as safe and effective, it will provide a significant 'Level 1' treatment option which may help sufferers of anxiety and provide significant support to use in a clinical setting. It may also ease concerns about the potential reinstitution to restricted markets, which would provide socioeconomic benefit to poorer Pacific Island nations.

\section{Trial status}

Recruitment to commence September 2015.

\section{Abbreviations}

ACC: anterior cingulate cortex; AE: adverse event; ANOVA: analysis of variance; ASEX: Arizona Sexual Experiences Scale; BAl: Beck Anxiety Inventory; CRF: case report form; DSM-IV: Diagnostic and Statistical Manual of Mental Disorders - Fourth Edition; GABA: gamma-aminobutyric acid; GAD: generalised anxiety disorder; HAM-A: Hamilton Anxiety Rating Scale; IAPS: International Affective Picture System; MADRS: Montgomery-Åsberg Depression Rating Scale; MINI-6.0: Mini International Neuropsychiatric Interview Version 6.0; MMRM: Mixed-effects model, repeated measures; MRS: magnetic resonance spectroscopy; NAA: N-acetyl-I-aspartate; NHMRC: National Health Medical Research Council; PSWQ: Penn State Worry Questionnaire; RCT: randomised clinical trial; SAFTEE: Systematic Assessment for Treatment of Emergent Effects; SIGH-A: Structured Interview Guide for the Hamilton Anxiety Rating Scale; SIGMA: Structured Interview Guide for the Montgomery-Åsberg Depression Rating Scale; SNP: single nucleotide polymorphism; SPSS: Statistical Package for Social Sciences software; SRRS: Social Readjustment Rating Scale; WHOQOL-BREF: World Health Organization Quality of Life - BREF. 


\section{Competing interests}

JS has received honoraria, research support, royalties, or consultancy or travel grant funding from Integria Health (who is co-funding the study), Blackmores, Bioceuticals, Taki Mai (a kava-producing and selling company), Pepsico, HealthEd, Soho-Flordis, Pfizer, Elsevier, the Society for Medicinal Plant and Natural Product Research, CR Roper Fellowship, and the National Health Medical Research Council (NHMRC).

\section{Authors' contributions}

KMS and JS contributed to the conception and design of the study and wrote the manuscript. CS and CB contributed to the imaging and genetics design of the study, respectively. CB, CS, CKS, AS, RT, GJB, JM, ST, RT, CX, PM, and $\mathrm{MH}$ provided technical advice and contributed to a critical review of the manuscript. JS as the principal investigator provided a critical review and edit of the manuscript. All authors read and approved the final manuscript.

\section{Acknowledgments}

This grant is funded by a National Health and Medical Research Council project grant (APP1063383) and is co-sponsored by MediHerb (Integria Healthcare (Australia) Pty. Ltd). Dr Jerome Sarris is supported by a CR Roper Fellowship. Chad Bousman is supported by a Ronald Phillip Griffith Fellowship. Karen Savage is supported by an APA national scholarship and by an NHMRC grant bursary (APP1063383).

\section{Author details}

${ }^{1}$ The University of Melbourne, Department of Psychiatry, The Melbourne Clinic, Melbourne, Australia. ${ }^{2}$ Swinburne University of Technology, Centre for Human Psychopharmacology, Swinburne, Australia. ${ }^{3}$ Department of Psychiatry, The University of Queensland, Melbourne, Australia. ${ }^{4}$ Department of Psychiatry, The University of Melbourne, Parkville, Australia. ${ }^{5}$ Florey Institute of Neuroscience and Mental Health, The University of Melbourne, Parkville, Australia. ${ }^{6}$ Department of General Practice, The University of Melbourne, Parkville, Australia. ${ }^{7}$ Brain and Mental Health Laboratory, School of Psychological Science, Monash University, Monash, Australia. ${ }^{8}$ Swinburne University of Technology, Brain and Psychological Sciences Centre, Swinburne, Australia. ${ }^{9}$ School of Psychological Science, Monash University, Monash, Australia. ${ }^{10}$ Department of Internal Medicine II, Section of Gastroenterology and Hepatology, Klinikum Hanau, Teaching Hospital of the Johann Wolfgang Goethe University of Frankfurt/Main, Frankfurt, Germany. ${ }^{11}$ Department of Medicinal Chemistry, College of Pharmacy, University of Minnesota, Duluth, USA.

Received: 29 April 2015 Accepted: 30 September 2015

Published online: 02 November 2015

\section{References}

1. Stein D. Generalised anxiety disorder. Rethinking diagnosis and rating. CNS Spectr. 2005;10(12):930-4.

2. American Psychiatric Association (APA). Diagnostic and statistical manual of mental disorders. Washington, DC: American Psychiatric Association; 2013.

3. Baxter AJ, Scott KM, Vos T, Whiteford HA. Global prevalence of anxiety disorders: a systematic review and meta-regression. Psychol Med. 2013 May;43(5):897-910.

4. Wittchen HU, Jacobi F, Rehm J, et al. The size and burden of mental disorders and other disorders of the brain in Europe. Eur Neuropsychopharmacol. 2011;21(9):655-79.

5. McEvoy PM, Grove R, Slade T. Epidemiology of anxiety disorders in the Australian general population: findings of the 2007 Australian National Survey of Mental Health and Wellbeing. Aust N Z J Psychiatry. 2011;45(11):957-67.

6. Department of Health and Aging. The mental health of Australians: anxiety disorders in Australia. 2012.

7. Hoffman EJ, Mathew SJ. Anxiety disorders: a comprehensive review of pharmacotherapies. Mt Sinai J Med. 2008;75(3):248-62.

8. Hidalgo RB, Tupler LA, Davidson JR. An effect-size analysis of pharmacologic treatments for generalized anxiety disorder. J Psychopharmacol. 2007;21(8):864-72.

9. Papakostas G. Tolerability of modern antidepressants. J Clin Psychiatry. 2008;69 Suppl 1:8-13.

10. Rickels K, Rynn M. Pharmacotherapy of generalized anxiety disorder. J Clin Psychiatry. 2002;63 Suppl 14:9-16.
11. Baldwin DS, Ajel Kl, Garner M. Pharmacological treatment of generalized anxiety disorder. Curr Top Behav Neurosci. 2010;2:453-67.

12. Cuijpers P, Sijbrandij M, Koole S, Huibers M, Berking M, Andersson G. Psychological treatment of generalized anxiety disorder: a meta-analysis. Clin Psychol Rev. 2014;34:130-40.

13. Hunot V, Churchill R, Teixeira V, Silva de Lima M. Psychological therapies for generalised anxiety disorder (Review). Cochrane Database of Systematic Reviews. 2007; 24:(1):CD001848

14. Gunter RW, Whittal ML. Dissemination of cognitive-behavioral treatments for anxiety disorders: Overcoming barriers and improving patient access. Clin Psychol Rev. 2010;30(2):194-202.

15. Weisberg RB, Dyck I, Culpepper L, Keller MB. Psychiatric treatment in primary care patients with anxiety disorders: a comparison of care received from primary care providers and psychiatrists. Am J Psychiatry. 2007;164(2):276-82.

16. Goldberg D. Epidemiology of mental disorders in primary care settings. Epidemiol Rev. 1995;17:182-90.

17. Koerner $\mathrm{N}$. The importance of taking an integrative approach to the study of worry, intolerance of uncertainty, and generalized anxiety disorder. Can Psychol/Psychol Can. 2014;55(1):44-7.

18. Starcevic V, Portman ME, Beck AT. Generalized anxiety disorder: between neglect and an epidemic. J Nervous Mental Dis. 2012;200:664-7.

19. Lebot V, Merlin M, Lindstrom L. Kava: the Pacific drug. New Haven, Connecticut: Yale University Press; 1992

20. Singh YN. Kava: an overview. J Ethnopharmacol. 1992;37(1):13-45.

21. Mathews JM, Etheridge AS, Valentine $J$, et al. Pharmacokinetics and disposition of the kavalactone kawain: interaction with kava extract and kavalactones in vivo and in vitro. Drug Metab Dispos. 2005;33:1555-63.

22. Holm E, Staedt U, Hepp J, et al. Untersuchungen zum Wirkungsprofil von D, L-kavain. Arzneim Forsch. 1991;41(7):673-83.

23. Magura El, Kopanitsa MV, Gleitz J, et al. Kava extract ingredients, (+)-methysticin and (+/-)-kavain inhibit voltage-operated $\mathrm{Na}(+)$-channels in rat CA1 hippocampal neurons. Neuroscience. 1997;81(2):345-51.

24. Gleitz J, Beile A, Peters T. (+/-)-Kavain inhibits veratridine-activated voltagedependent $\mathrm{Na}(+)$-channels in synaptosomes prepared from rat cerebral cortex. Neuropharmacol. 1995;34(9):1133-8.

25. Martin HB, McCallum M, Stofer WD, et al. Kavain attenuates vascular contractility through inhibition of calcium channels. Planta Med. 2002;68(9):784-9.

26. Walden J, von Wegerer J, Winter $U$, et al. Effects of kawain and dihydromethysticin on field potential changes in the hippocampus. Prog Neuropsychopharmacol Biol Psychiatry. 1997;21(4):697-706.

27. Jussofie A, Schmiz A, Hiemke C. Kavapyrone enriched extract from Piper methysticum as modulator of the GABA binding site in different regions of rat brain. Psychopharmacology (Berl). 1994;116(4):469-74.

28. Uebelhack R, Franke L, Schewe HJ. Inhibition of platelet MAO-B by kava pyrone-enriched extract from Piper methysticum Forster (kava-kava). Pharmacopsychiatry. 1998;31(5):187-92.

29. Wu D, Yu L, Nair M, DeWitt D, et al. Cyclooxygenase enzyme inhibitory compounds with antioxidant activities from Piper methysticum (kava kava) roots. Phytomedicine. 2002;9:41-7.

30. Baum SS, Hill R, Rommelspacher $\mathrm{H}$. Effect of kava extract and individual kavapyrones on neurotransmitter levels in the nucleus accumbens of rats. Prog Neuropsychopharmacol Biol Psychiatry. 1998;22(7):1105-20.

31. Seitz U, Schule A, Gleitz J. [3H]-monoamine uptake inhibition properties of kava pyrones. Planta Med. 1997;63(6):548-9.

32. Thompson R, Ruch W, Hasenohrl RU. Enhanced cognitive performance and cheerful mood by standardized extracts of Piper methysticum (Kava-kava). Hum Psychopharmacol. 2004;19(4):243-50.

33. Sarris J, Scholey A, Schweitzer I, et al. The acute effects of kava and oxazepam on anxiety, mood, neurocognition, and genetic correlates: a randomized, placebo-controlled, double-blind study. Hum Psychopharmacol. 2012;27(3):262-9.

34. Pittler $\mathrm{MH}$, Ernst EE. Efficacy of kava extract for treating anxiety: systematic review and meta-analysis. J Clin Psychopharm. 2000;20(1):84-9.

35. Sarris J, Laporte E, Schweitzer I. Kava: a comprehensive review of efficacy, safety, and psychopharmacology. Aust N Z J Psychiatry. 2011;45(1):27-35.

36. Sarris J, Kavanagh D, Byrne G, Bone K, Adams J, Deed G. The Kava Anxiety Depression Spectrum Study (KADSS): a randomized, placebo-controlled, cross-over trial using an aqueous extract of Piper methysticum. Psychopharmacol. 2009;205(3):399-407. 
37. Sarris J, Stough C, Bousman C, et al. Kava in the treatment of generalized anxiety disorder: a double-blind, randomized, placebo-controlled study. J Clin Psychopharmacol. 2013;33(5):643-8.

38. Hamilton M. The assessment of anxiety states by rating. $\mathrm{Br} J$ Med Psychol. 1959;32:50-5.

39. Beck AT, Epstein N, Brown G, Steer RA. An inventory for measuring clinical anxiety: psychometric properties. J Consult Clin Psychol. 1988;56(6):893-7.

40. Meyer TJ, Miller ML, Metzger RL, Borkovec TD. Development and validation of the Penn State Worry Questionnaire. Behav Res Ther. 1990;28:487-95.

41. Montgomery SA, Asberg M. A new depression scale designed to be sensitive to change. Br J Psychiatry. 1979;134(4):382-9.

42. Skevington SM, Lofty M, O'Connell KA. The World Health Organization's WHOQOL-BREF quality of life assessment: psychometric properties and results of the international field trial. A report from the WHOQOL group. Qual Life Res. 2004;13(2):299-310.

43. Holmes TH, Rahe RH. The Social Re-adjustment Rating Scale. J Psychosom Res. 1967;11:213-8.

44. Kessler RC, Andrews G, Colpe LJ, et al. Short screening scales to monitor population prevalences and trends in non-specific psychological distress. Psychol Med. 2002;32:959-976.

45. McGahuey CA, Gelenberg AJ, Laukes CA, et al. The Arizona Sexual Experience Scale (ASEX): reliability and validity. J Sex Marital Ther. 2000;26:25-40.

46. Lecrubier $Y$, Sheehan D, Weiller E, Amorim $\mathrm{P}$, Bonora I, Sheehan $\mathrm{K}$, et al. The M.I.N.I. International Neuropsychiatric Interview (M.I.N.I.). A short diagnostic structured interview: reliability and validity according to the CIDI. Eur Psychiatry. 1997;12:224-31.

47. Shear MK, Vander Bilt J, Rucci P, Endicott J, Lydiard B, Oto MW, et al. Reliability and validity of a structured interview guide for the Hamilton Anxiety Rating Scale (SIGH-A). Depress Anxiety. 2001;13:166-78.

48. Maier W, Buller R, Philipp M, Heuser I. The Hamilton Anxiety Scale: reliability, validity and sensitivity to change in anxiety and depressive disorders. J Affect Disord. 1988;14:61-8.

49. Brown TA, Antony MM, Barlow DH. Psychometric properties of the Penn State Worry Questionnaire in a clinical anxiety disorders sample. Behav Res Ther. 1992;30:33-7.

50. Williams JBW, Kobak KA. Development and reliability of a structured interview guide for the Montgomery-Asberg Depression Rating Scale (SIGMA). Br J Psychiatry. 2008;192(1):52-8.

51. Gustavsson JP, Bergman H, Edman G, Ekaselius L, von Knorring L, Linder J. Swedish universities Scales of Personality (SSP) - Manual 2.0. Karolinska Institute \& Uppsala University, Stockholm, Sweden. 2000.

52. Lang PJ, Bradley MM, Cuthbert BN. International Affective Picture System (IAPS): affective ratings of pictures and instruction manual. Technical Report A-8. Gainesville, FL: University of Florida; 2008.

53. Spielberger CD, Gorsuch RL, Lushene R, Vagg PR, Jacobs GA. Manual for the State-Trait Anxiety Inventory. Palo Alto, CA: Consulting Psychologists Press; 1983.

54. Rae CD. A guide to the metabolic pathways and function of metabolites observed in human brain 1-H magnetic resonance spectra. Neurochem Res. 2014;39:1-36.

55. IBM SPSS Statistics for Windows, Version 22.0. Armonk, NY: IBM Corp; 2013.

56. Uppman M. The reliability of GABA measurements in the human brain by MRS at 3T. 2010

57. Heberlein K, Brown MA. Spectral editing with SVS. Applications Guide. Munich, Germany: Siemens Corporation; 2009.

58. Wood SJ, Kennedy D, Phillips LJ, Seal ML, et al. Hippocampal pathology in individuals at ultra-high risk for psychosis: a multi-modal magnetic resonance study. Neuroimage. 2010;52:62-8.

59. Thompson CM, Shure L. MathWorks. Bioinformatics Toolbox: User's Guide (R2012a). Mathworks:Massachusetts, USA; 1995.

60. Berger GE, Wood SJ, Wellard RM, et al. Ethyl-eicosapentaenoic acid in first episode psychosis. A 1H-MRS study. Neuropsychopharmacology. 2008;33(10):2467.

61. Ernst E. A re-evaluation of kava (Piper methysticum). Br J Clin Pharmacol. 2007;64(4):415-7.

62. Teschke R. Kava hepatotoxicity - a clinical review. Ann Hepatol. 2010;9(3):251-5.

63. Sarris J, Teschke R, Stough C, et al. Re-introduction of kava (Piper methysticum) to the EU: is there a way forward? Planta Med. 2011;77:107-10.
64. Coulter D. Assessment of the risk of hepatotoxicity with kava products. WHO appointed committee. Geneva: World Health Organization; 2007.

65. Levine J, Schooler N. SAFTEE (Systematic Assessment for Treatment of Emergent Events). A new technique for detecting side effects in clinical trials. Clin Neuropharmacol. 1984;7(1):861-77.

66. Chan A, Tetzlaff JM, Altman DG, Laupacis A, Gøtzsche PC, Krleža-Jerić K, et al. SPIRIT 2013 Statement: defining standard protocol items for clinical trials. Ann Intern Med. 2013;158:200-7.

67. Rothman DL, Hyder F, Sibson N, et al. In vivo magnetic resonance spectroscopy studies of the glutamate and GABA neurotransmitter cycles and functional neuroenergetics. In: Davis KL, Charney D, Coyle JT, Nemeroff $C B$, editors. Neuropsychopharmacology: the fifth generation of progress. American College of Neuropsychopharmacology. 2002.

68. Puts NAJ, Edden RAE. In vivo magnetic resonance spectroscopy of GABA: a methodological review. Prog Magn Reson Spectrosc. 2012;60:29-41.

69. Etkin A, Wager TD. Functional neuroimaging of anxiety: a meta-analysis of emotional processing in PTSD, social anxiety disorder, and specific phobia. Am J Psychiatry. 2007;164:10.

70. Mullins PG et al. Current practice in the use of MEGA-PRESS spectroscopy for the detection of GABA. Neuroimage. 2013;86:43-52.

71. Allman JM, Hakeem A, Erwein JM, Nimchinsky E, Hof P. The anterior cingulate cortex. The evolution of an interface between emotion and cognition. Ann N Y Acad Sci. 2001;935:107-17.

72. Bush G, Luu P, Posner MI. Cognitive and emotional influences in anterior cingulate cortex. Trends Cogn Sci. 2000;4:215-22.

73. Critchley HD, Mathias CJ, Josephs O, O'Doherty J, Zanini S, Dewar BK, et al. Human cingulate cortex and autonomic control: converging neuroimaging and clinical evidence. Brain. 2003;126:2139-52.

74. Etkin A, Prate KE, Hoeft F, Menon V, Schatzberg AF. Failure of anterior cingulate activation and connectivity with the amygdala during implicit regulation of emotional processing in generalised anxiety disorder. Am J Psychiatry. 2010;167:545-54.

75. Luu P, Pederson SM. The anterior cingulate cortex: regulating actions in context. In: Posner Ml, editor. Cognitive neuroscience of attention. New York: Guilford Publication Inc; 2004.

76. Murphy FC, Nimmo-Smith I, Lawrence AD. Functional neuroanatomy of emotions: a meta-analysis. Cogn Affect Behav Neur. 2003;3(3):207-33.

77. Schienle A, Hettema JM, Caceda R, Nemeroff CB. Neurobiology and genetics of generalised anxiety disorder. Psychiatr Ann. 2011;41(2):113-23.

78. Vogt BA, Finch DM, Olson CR. Functional heterogeneity in cingulate cortex: the anterior executive and posterior evaluative regions. Cereb Cortex. 1992;2(6):435-43.

79. Wegner DM, Schneider DJ, Carter SL, White TL. Paradoxical effects of thought suppression. J Pers Soc Psychol. 1987;63:903-12.

\section{Submit your next manuscript to BioMed Central and take full advantage of:}

- Convenient online submission

- Thorough peer review

- No space constraints or color figure charges

- Immediate publication on acceptance

- Inclusion in PubMed, CAS, Scopus and Google Scholar

- Research which is freely available for redistribution 